\title{
High-G and High Bandwidth Characterization of Piezoresistive MEMS Accelerometers for Crash Test Applications
}

\author{
Udo Lang ${ }^{1}$, Raphael Theurillat ${ }^{3}$, Stéphane Kühne ${ }^{2}$, Thomas Kiko², Remco Leine ${ }^{3}$, Claudio Cavalloni ${ }^{2}$ \\ ${ }^{1}$ Lucerne University of Applied Sciences and Arts, CC Mechanical Systems, 6048 Horw, Switzerland \\ ${ }^{2}$ Kistler Instrumente AG, Eulachstr. 22, 8408 Winterthur, Switzerland, stephane.kuehne@kistler.com \\ ${ }^{3}$ Center of Mechanics, Department of Mechanical and Process Engineering, 8092 Zurich, Switzerland
}

\begin{abstract}
Crash and pyro shock tests, which are used to improve or test technical safety structures, are represented by high $\mathrm{g}$ and high bandwidth excitations. Accelerometers used to record these tests must be able to closely capture data under extreme conditions without major distortions. Therefore, reliable calibration schemes have to be developed that allow for a sensor characterization over the whole measurement range. Existing approaches are either restricted in bandwidth or in amplitude. In the current paper, we present a methodology that can be applied to typical MEMS accelerometers based on air gap damping and that considerably improves sensor accuracy even at high $\mathrm{g}$ and high bandwidth ranges.
\end{abstract}

Key words: Piezoresistive accelerometer, Kalman filter, high-g impact, crash test.

\section{Introduction}

In automobile crash tests piezoresistive accelerometers are required by legal regulations. Furthermore, for certain applications in such crash tests, the sensors need to be critically damped, i.e. they have to exhibit a damping ratio of 0.7 to protect the sensors from ringing when the seismic mass hits the end of measurement ranges during the high-g shock events. For MEMS sensors, this damping ratio is commonly achieved by gas damping in small gaps and is called squeeze film damping. The shock events during crash tests lead to accelerations of up to several thousands of $\mathrm{g}$. At the same time, a crash event represents a high bandwidth excitation of the mechanical measuring system. For a defined interpretation of the measurement results, a validated mechanical model of the sensor and its mounting is necessary. A common high $\mathrm{g}$ characterization method is the Split Hopkinson bar [1]. While this method allows for high $\mathrm{g}$ application, the bandwidth is restricted to the natural frequency of the longitudinal wave propagation in the bar. On the other hand, a system characterization on a shaker allows to examine a broader frequency spectrum but is restricted to low excitation amplitudes. In order to capture the high $\mathrm{g}$ and high bandwidth signals typical for crash tests, other methodo- logies are necessary to fully characterize and calibrate the dynamic sensor behavior.

In this paper, which is based on [2], we present a model-based methodology to characterize the sensor response to high $\mathrm{g}$ and high bandwidth excitations. The characterized device type is a piezoresistive accelerometer M126AM0C7 from Kistler Instrumente AG. Its critical damping ratio is achieved by small gap air damping shown in the schematic cross-section in Figure 1. A hammer blow on the metal base plate, to which the sensor is attached, is used to achieve a high impact broad bandwidth excitation. By measuring the actual movements of the device under test (DUT) with a laser interferometer, the real excitation independent of the accelerometer mounting to the base plate is precisely known. For this reason, the use of laser interferometers for the characterization of shock events has been implemented in testing environments [3].

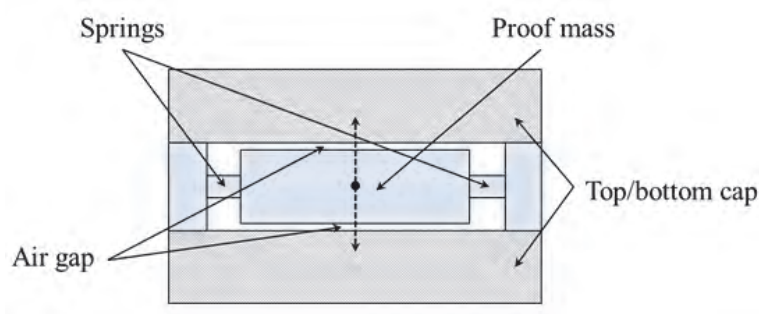

Fig. 1: Schematic cross-section of the piezoresistive MEMS accelerometer Kistler M126AM0C7. 
As squeeze-film damping at high $\mathrm{g}$ excitations is a complex and nonlinear process [4], we then introduce a mechanical, non-linear two degrees of freedom model to fully capture the complex nature of the mechanical oscillations. Its main difference from standard literature models is that the air is treated as second oscillating mass with its own degree of freedom (DOF). The experimental data simultaneously obtained from the laser interferometer measurement as well as from the recorded accelerometer output signal is then used for the system parameter identification and model validation. This process is based on the application of a nonlinear augmented unscented Kalman filter [5] to the sampled data. Thereby, the sensor behavior can be fully characterized over a large excitation range and more precise high shock calibration and measurements become feasible.

\section{Experimental}

The calibration of the system parameters is achieved by a sophisticated experimental setup and the application of a nonlinear augmented unscented Kalman filter to the sampled data. Firstly, the accelerometer is mounted to a metal base plate and its response to hammer blows on the metal plate recorded. At the same time, a Laser vibrometer is used to measure the base point movements of the metal plate as well as the DUT housing (see Figures 2 and 3 ). Therefore, mounting effects (e.g. difference between use of adhesive tape and mechanical screws) do not have to be considered further. Typical experimental results obtained with this setup are shown in Figure 4. In order to reduce the noise of the vibrometer velocity data the "Smoothing by Spline Functions" implemented in Matlab [6] is used. Thus, a continuous differentiable velocity signal is obtained to compute the reference acceleration while keeping all relevant information (Fig.4a)).

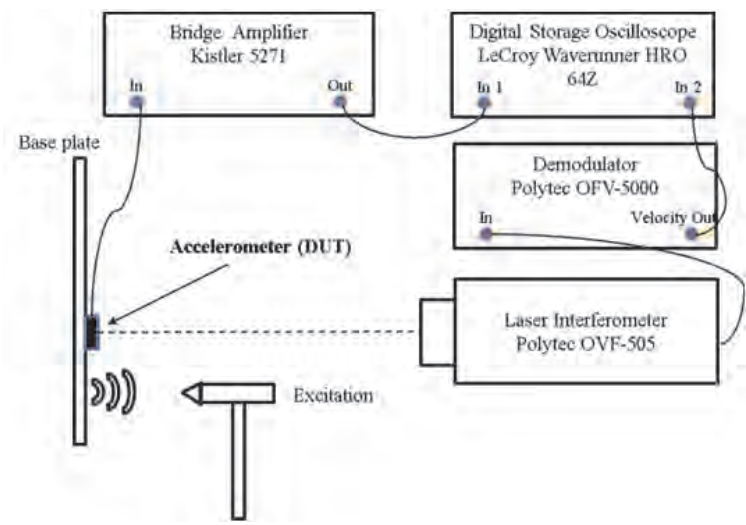

Fig. 2. Experimental setup: excitation by hammer blows on a metal base plate and recording of the device under test (DUT) output response as well as the accelerometer housing movement by Laser interferometer.

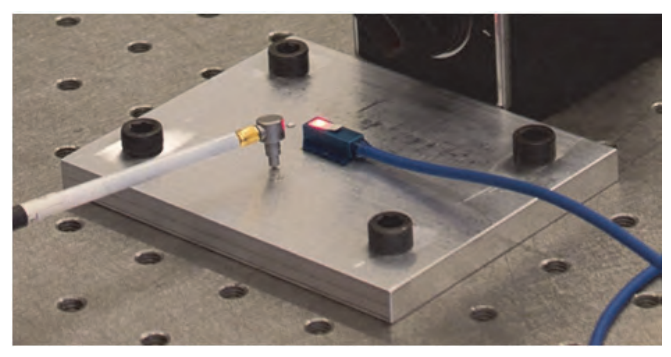

Fig. 3: Actual test setup: hammer, base plate and piezoresistive accelerometer Kistler M126AM0C7.

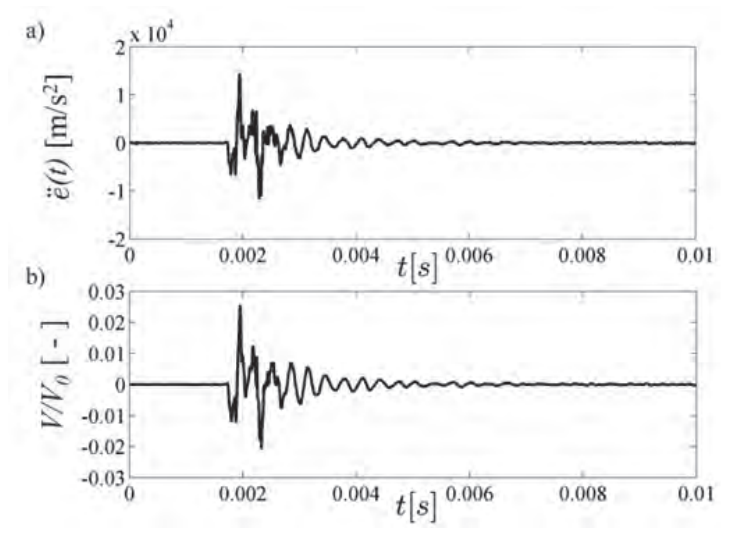

Fig. 4: a) Typical acceleration signal obtained for the DUT housing after processing and differentiating the vibrometer velocity signal, b) corresponding accelerometer output signal.

\section{Dynamic Model and Parameter Estimation}

The commonly accepted model for accelerometers assumes a linear relationship between acceleration and output signal. This implies a constant damping independent of excitation frequencies or amplitudes. However, this assumption is not valid for air damping in a narrow gap where large proof mass deflections and a broad bandwidth excitation occur. As the dynamic relative movements in the air gap during shock events lead to very complex flow processes, this relatively simple model cannot describe the accelerometer response to excitation as commonly experienced in crash or pyro blast tests. Due to the highly complex and nonlinear nature of the air damping process during shock events every single MEMS sensor of every manufacturer with this damping concept will exhibit different damping behavior caused by slightest production fluctuations.

Therefore, it is necessary to calibrate every sensor with high $g$ and high bandwidth excitations to provide the experimentalist with a sensor whose behavior is known in the whole measurement range. The approach shown herein using the presented experimental setup combined with a model-based system parameter estimation by an unscented Kalman filter (UKF) is a method suitable to calibrate acceleration sensors. 
Firstly, a more detailed mechanical model of the sensor has been developed (Figure 5). The connection of the proof mass $m_{1}$ to the accelerometer housing with the displacement excitation $e(t)$ is modeled through a non-linear force element sensitive to both displacement $y(t)$ and velocity $\dot{y}(t)$ as well as their products, i.e.

$$
\begin{gathered}
f_{1}=c_{1} y+c_{2} \dot{y}+c_{3}|y| y+c_{4}|y| \dot{y}+c_{5}|\dot{y}| y+ \\
c_{6}|\dot{y}| y+c_{7} y^{3}+c_{8} y^{2} \dot{y}+c_{9} \dot{y}^{2} y+c_{10} \dot{y}^{3}
\end{gathered}
$$

Therefore, $f_{l}$ is a combined non-linear springdamper-element. As a further refinement, a mass $m_{2}$ is attributed to the air in the gap. This air mass $m_{2}$ is in turn connected to both the proof mass and to the accelerometer housing by the two linear spring-damper elements $f_{2}\left(c_{21}, c_{22}\right)$ and $f_{3}\left(c_{31}, c_{32}\right)$. The equations of motion in state space first order form yields:

$$
\begin{aligned}
& \frac{d}{d t}\left(\begin{array}{c}
y_{1} \\
y_{2} \\
\dot{y}_{1} \\
\dot{y}_{2}
\end{array}\right)= \\
& \left(\begin{array}{c}
\dot{y}_{1} \\
\dot{y}_{2} \\
-\frac{1}{m_{1}}\left(f_{1}\left(y_{1}, \dot{y}_{1}\right)+f_{3}\left(y_{1}-y_{2}, \dot{y}_{1}-\dot{y}_{2}\right)\right)-\ddot{e} \\
-\frac{1}{m_{2}}\left(f_{2}\left(y_{2}, \dot{y}_{2}\right)-f_{3}\left(y_{1}-y_{2}, \dot{y}_{1}-\dot{y}_{2}\right)\right)-\ddot{e}
\end{array}\right)(2) .
\end{aligned}
$$

The output signal $v(t)$ of the sensor becomes

$$
v(t)=k y_{1}(t)
$$

In the next step the unknown parameters of $f_{l}$, $f_{2}$ and $f_{3}$ need to be determined. This key step is achieved by an UKF based on the known accelerometer excitation and output $e(t)$ and $v(t)$, respectively. The Kalman filter predicts the state of the described system model and averages this prediction with a new measurement. A weighted average is used for which the weights are calculated from the covariance matrix, being a measure of the estimated uncertainty of the model-based prediction of the state. The Unscented Kalman Filter (UKF) extends the classical Kalman filter to nonlinear systems and avoids the use of Jacobians by using the unscented transform [7].

The procedure of the UKF can roughly be described as follows:

- there exists a current estimation of the state of a system

- in a prediction step the mean and the standard deviation of the state are calculated based on the physical model of the system
- in an update step this prediction is compared to actual measurements and the estimation of the state of the system is improved.

Herein, an implementation of the UKF in Matlab [6] is used to estimate the system parameters $c_{i}$ of equations (1) and (2). Figure 5 illustrates the improvement of the parameter estimation during the application of the UKF routine to a recorded impulse response shown in Fig.5a). The frequency response of the model from the initial state (Fig.5b)) and after the UKF estimation (Fig.5c)) are compared to the measured response of the piezoresistive accelerometer.

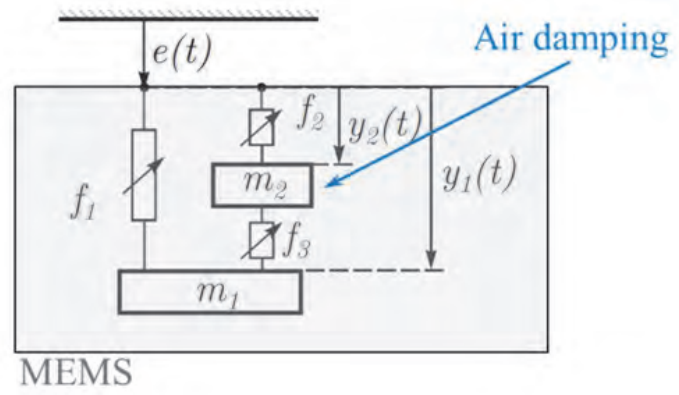

Fig. 5. Dynamic model of the MEMS accelerometer: proof mass $m_{1}$ with additional air mass $\mathrm{m}_{2}$ and spring-damper elements $\left(f_{l-3}\right)$, displacement excitation e $(t)$, movements of the proof mass and air mass relative to the accelerometer housing are denoted as $y_{1}(t)$ and $y_{2}(t)$, respectively.

a)

b)
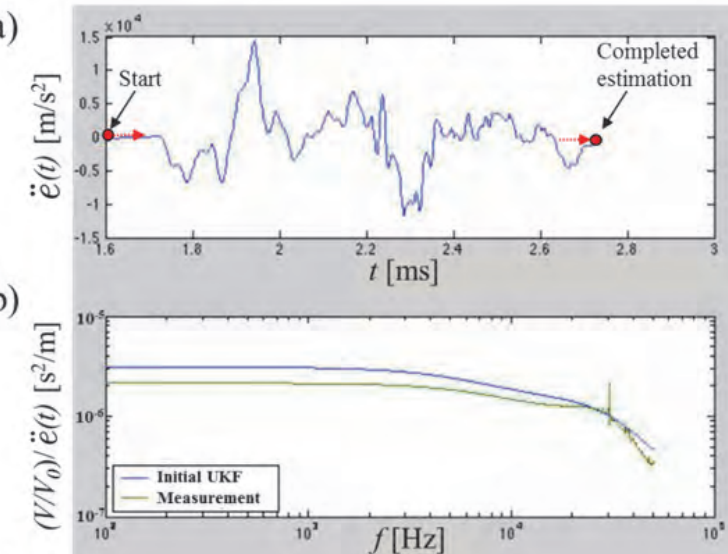

c)

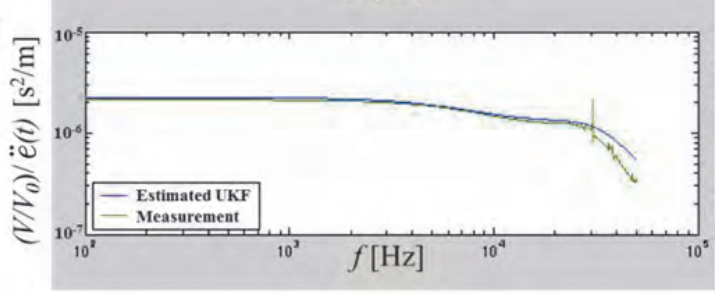

Fig. 6. Unscented Kalman Filter (UKF) parameter estimation based on measurement data: a) impulse response of measurement, $b$ ) frequency response of initial UKF, c) frequency response of estimated UKF. 

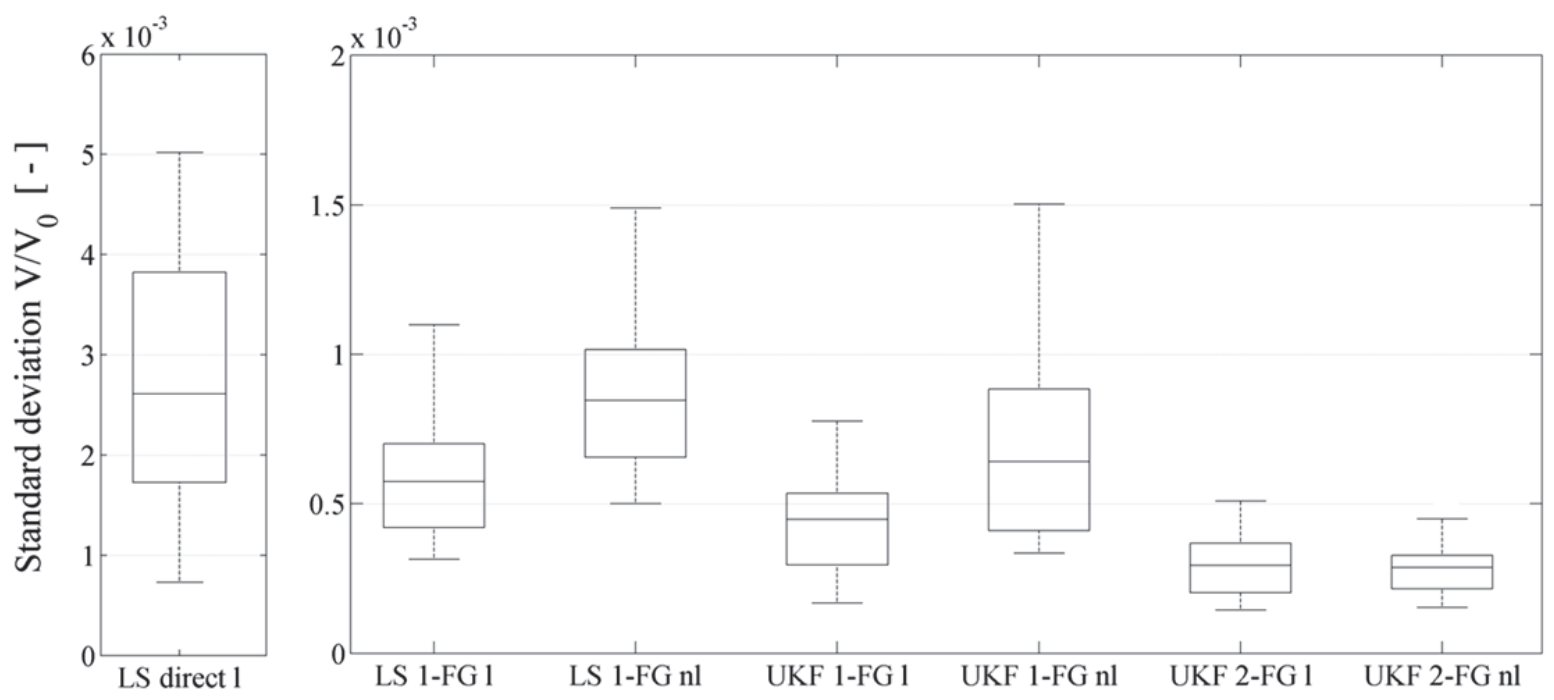

Fig. 7. Comparison of standard deviation between measurement data and simulated sensor response for a system excitation with high amplitude impulse.

\section{Model validation}

For the model validation, excitation signal data sets differing from the ones for the parameter estimation are used as input signal for simulations. The comparison of the simulated data and the measured sensor output data is then used to validate the model and quantify its accuracy. Seven different models are compared:

- LS direct 1: standard model with direct proportional output estimated by least square method (LS).

- LS 1-FG 1: standard one degree of freedom model with linear spring-damper element and parameter estimation by LS.

- LS 1-FG nl: standard one degree of freedom model with nonlinear spring-damper element, parameter estimation by LS.

- UKF 1-FG 1: standard one degree of freedom model with linear spring-damper element, parameter estimation by UKF.

- UKF 1-FG nl: standard one degree of freedom model with nonlinear spring-damper element, parameter estimation by UKF.

- UKF 2-FG 1: two degrees of freedom model with linear spring-damper element and parameter estimation by UKF.

- UKF 2-FG nl: two degrees of freedom model with nonlinear spring-damper element (Fig.4) and parameter estimation by UKF.

The results of the evaluation are summarized in Figure 7. The standard deviation between measured data and performed simulations is plotted for the models introduced above. The output standard deviation is normalized to the accelerometer supply voltage $\left(V / V_{0}, V_{0}=10 \mathrm{~V}\right)$. The boxplots show the deviation of 26 measurement and simulation datasets for each model type. The presented result for the UKF 2FG $\mathrm{nl}$ model yields a more than one order of magnitude smaller standard deviation than the commonly used standard direct LS model. The developed approach therefore represents an accurate method for modeling and estimating the behavior of piezoresistive MEMS accelerometers experiencing high $\mathrm{g}$ and high bandwidth excitations.

\section{Discussion}

The usual approaches to model squeeze film damping in literature are limited to small amplitude oscillations of the deflected structure and are therefore not suitable for describing MEMS accelerometers with air damping when exposed to shock events with high $\mathrm{g}$ and high bandwidth accelerations. Hence, a new mechanical nonlinear model had to be developed. At the same time, the currently used approaches for piezoresistive accelerometer calibration and testing do not represent the high $\mathrm{g}$ and high bandwidth excitation during shock events and thus the output signals obtained from sensors calibrated with standard procedures are resulting in large errors in this measurement range.

Therefore, the presented approach has two main advantages over the conventional ones: the experimental setup allows for the precise measurement of application-related excitations and for effective model-based system parameter identification. It was shown that this 
approach yielded the best approximation of the actual accelerometer behavior and is thus a very promising method for fast and reliable accelerometer calibration.

In the future, it will hence be possible to fully capture the nonlinear mechanical behavior of an individual sensor by determining the model parameters describing its nonlinear behavior. Accelerometers calibrated this way, would allow to precisely trace back the high $\mathrm{g}$ and high bandwidth excitation experienced in crash impacts. This represents a major methodical improvement compared to the current state-ofthe-art approach, where the combined high $\mathrm{g}$ and high bandwidth behavior of sensors used in crash or other high shock applications is usually not known.

\section{Conclusion}

A methodology based on a sophisticated experimental setup and a parameter estimation routine relying on a newly developed mechanical model and an unscented Kalman filter (UKF) was presented. This approach can fully capture the nonlinear mechanical behavior of an individual piezoresistive acceleration sensor. The method is suitable for model-based accelerometer calibration with high $\mathrm{g}$ and broad bandwidth excitation and thus permits shock event measurements with higher accuracy than so far achieved in science and industry.

\section{References}

[1] R. Martin, J.Letterneau, T. Kwa, Testing a HighSurvivability, High-Shock 20 '000 g Accelerometer, Part 1: Preliminary Prototype Testing, Sensor Magazine, http://www.sensorsmag.com/aerospace-militaryhs/testing-high-survivability-high-shock-20000-gaccelerometer-9613

[2] R. Theurillat, Theoretische und experimentelle Untersuchung piezoresistiver Beschleunigungssensoren, Masterthesis, ETH Zurich, 2012.

[3] NASA Technical Standard, NASA-STD-7003A, (2011).

[4] J. Blech, On isothermal squeeze films, Journal of Lubrication Technology, 105, 4 (1983), 615-620.

[5] J. Hartikainen, J., S. Särkkä, Optimal filtering with Kalman filters and smoothers a manual for Matlab toolbox ekf/ukf, Helsinki University of Technology, Espoo, Finland (2008).

[6] Matlab Version 2011b (7.13.0.564), The MathWorks Inc., Natick, Massachusetts (2011).

[7] SJ Julier, JK Uhlmann Unscented filtering and nonlinear estimation, Proceedings of the IEEE 92 401-422 (2004),

doi:10.1109/JPROC.2003.823141 